\title{
MODELS FOR THE EMISSION-LINE SPECTRA OF THE LOW-EXCITATION HERBIG-HARO OBJECTS
}

\author{
Michael A. Dopita ${ }^{1}$, Saul Caganoff ${ }^{1}$, Richard D. Schwartz ${ }^{2}$ and \\ Martin Cohen 3 \\ ${ }^{1}$ Mt. Stromlo and Siding Spring Observatories, Australian National University \\ 2 Department of Physics, University of Missouri, St. Louis \\ ${ }^{3}$ NASA-Ames Research Center and Radio Astronomy Laboratory, \\ University of California, Berkeley
}

\begin{abstract}
The class of Low-Excitation Herbig-Haro Objects are characterised by [SII] and [OI] lines which are comparable in strength to $\mathrm{H}$-Alpha, by [NI] lines that are comparable to H-Beta, relatively weak [NII] and [OII] lines; little or no [OIII] emission and a very strong blue-UV "excess".This blue and UV continuum in low-excitation $\mathrm{HH}$ Objects was noted as a problem by Brugel, Böhm and Mannery (1981), Ortalani and D'Odorico (1980) and Böhm, Böhm-Vitense and Brugel (1981). The first suggestion that it results from collisionally enhanced Hydrogen two-photon (2q) continuum was by Dopita (1981). The subsequent observations of Dopita, Binette and Schwartz (1982) proved that this was indeed the case. However, although very close correlations between this enhancement and the emission-line spectrum were found, a fair theoretical description could only be obtained for very youthful shock models with ages of order 30 years. However, there seems to be no reason why low excitation $\mathrm{HH}$ shocks should be much younger than the high excitation shocks.

It is clear that a collisional excitation of atomic hydrogen is a dominant mechanism. The régime in which the early models and the observations diverge is precisely at velocities for which the material entering the shock is no longer appreciably pre-ionised. A prerequisite to obtain a large $2 q$ enhancement is the existence of a zone with a substantial un-ionised fraction of Hydrogen. Ohtani (1980) has emphasised that the relaxation between electrons and ions could become important in low velocity (non-preionising) shocks. Indeed this process is important wherever the cooling of the electron gas by inelastic collisions becomes comparable with the energy gain through superelastic collisions, such as is the case in shock models for oxygen-rich gas (Itoh, 1981a,b; Dopita, Binette and Tuohy, 1984). In this case a zone with different ion and electron temperatures can be maintained provided that there is no collective heating of the electrons ( McKee 1974 ;Pravdo and Smith 1979).
\end{abstract}


Using our code MAPPINGS, we computed a set of models with no collective heating for a variety of shock velocities. Gas in the pre-shock zone is initially in ionisation and temperature equilibrium with starlight at $8000 \mathrm{~K}$ with a dilution factor of $10^{-12}$. The composition is solar, except for the refractory elements $\mathrm{Mg}$ and $\mathrm{Si}$, which had to be depleted, presumably the result of trapping on grains. The 'Best Fit' chemical composition is, by number: $\mathrm{H}: \mathrm{He}: \mathrm{C}: \mathrm{N}: \mathrm{O}: \mathrm{Ne}: \mathrm{Mg}: \mathrm{Si}: \mathrm{S}=$

$1: 0.1: 4.2 \mathrm{E}-4: 8.7 \mathrm{E}-5: 6.9 \mathrm{E}-4: 9.8 \mathrm{E}-5: 2.8 \mathrm{E}-6: 2.7 \mathrm{E}-6: 1.9 \mathrm{E}-5$.

The effect of electron-ion relaxation is to prevent the electron temperature rising above the point at which collisional ionisation losses are becoming important. The individual line flux ratios given in Table 1 are generally in very good agreement. The [NI]/[NII] is rather too high, which suggests that the charge exchange reaction rate may still wrong. The spectrum of HH43 includes the higher excitation knot HH43A.

TABLE 1: A COMPARISON OF A MODEL $\left(v_{s}=45 \mathrm{~km} / \mathrm{s}\right)$ WITH LOW-EXITATION H-H OBJECTS.

\begin{tabular}{|c|c|c|c|c|}
\hline Ion & Wavelength & Shock Model & HH43A-C & HH47 \\
\hline$\overline{\mathrm{CI}}]$ & 2326 & 124 & 258 & - \\
\hline [OII] & 2470 & 2.5 & 34 & - \\
\hline MgII & 2800 & 36 & 82 & - \\
\hline $\mathrm{CI}]$ & 2968 & 163 & 77 & \\
\hline [OII] & $3727 / 29$ & 156 & 113 & $\overline{87}$ \\
\hline [SII] & $4068 / 76$ & 43.6 & 68.5 & 81 \\
\hline HI & 4101 & 20.0 & 16.1 & 20.4 \\
\hline HI & 4340 & 40.4 & 40.6 & 43.7 \\
\hline $\mathrm{MgI}]$ & $4562 / 71$ & 40.9 & 15.2 & 39.0 \\
\hline $\mathrm{H} \beta$ & 4861 & 100 & 100 & 100 \\
\hline [OIII] & 4959 & 0.0 & 2.1 & - \\
\hline [OIII] & 5007 & 0.0 & 6.4 & \\
\hline [NI] & $5198 / 200$ & 171 & 111 & 127 \\
\hline [OI] & 6300 & 374 & 340 & 320 \\
\hline$[\mathrm{OI}]$ & 6363 & 123 & 110 & 101 \\
\hline [NII] & 6548 & 4.9 & 30 & 26 \\
\hline $\mathrm{HI}$ & 6563 & 405 & 437 & 463 \\
\hline [NII] & 6584 & 14.5 & 90.1 & 67 \\
\hline [SII] & 6717 & 521 & 343 & 448 \\
\hline [SII] & 6731 & 475 & 326 & 471 \\
\hline \multicolumn{2}{|c|}{$\mathrm{F}(2 \mathrm{q}) / \mathrm{F}(\mathrm{HB})$} & 97.9 & 123 & 145 \\
\hline
\end{tabular}

\section{References}

Böhm,K.H., Böhm-Vitense,E.and Brugel,E.W.1981.Astrophys.J. (Lett.) 245 ,L113 Brugel, E.W., Böhm, K.H. and Mannery, E. 1981. Astrophys.J. 243,874 Dopita, M.A. 1981. in Exploring the Universe, Ed. F.D.Kahn, Dordrecht;Reidel Dopita, M.A., Binette,L. and Schwartz, R.D. 1982. Astrophys.J. 261, 183 Dopita, M.A., Binette, L. and Tuohy, I.R. 1984. Astrophys. J. 282, 142 Itoh,H. 1981a. Publ. Ast. Soc. Japan 33, 121 1981b. Publ. Ast. Soc. Japan 33, 521

$\overline{\mathrm{McKe}}$, C.F. 1974. Astrophys.J. 188,335

Ohtani,H. 1980. Publ. Ast. Soc. Japan 32, 11 Ortolani, S. and D'Odorico, S. 1980. Astron. Astrophys. 83, L8 Pravdo, S.H. and Smith, B.W. 1979. Astrophys.J. (Lett.) 234, L195 Schwartz, R.D., Dopita, M.A. amd Cohen, M. 1985. Astron. J. 90, 1820 